\title{
Systèmes de transport pour un développement intégré de l'Afrique : vers un réseau routier et ferroviaire panafricain
}

José Mvuezolo Bazonzi*

\begin{abstract}
Résumé
L'Afrique est un continent immense dont plusieurs contrées sont encore enclavées à ce jour. De ce fait, elle nécessite des systèmes de transport adéquats, efficaces et fort variés, susceptibles d'accélérer son développement. En effet, le manque de communication adéquate entre les zones de production et celles de forte densité et consommation est à l'origine d'un déséquilibre profond et pathologique entre l'offre et la demande globale des biens et services à travers l'espace continental. À côté du transport aérien fort onéreux, il s'avère opportun d'investir dans le transport routier et ferroviaire, plus accessible à la majorité de la population et véritable catalyseur de l'intégration et du développement du continent africain. Ainsi, dans le but de contribuer à la réflexion sur les alternatives au développement de l'Afrique, il nous semble nécessaire de jeter les bases d'une esquisse théorique du réseau routier et ferroviaire 'panafricain', dont le but serait de relier les grandes aires économiques et démographiques du continent afin de développer l'unité et la solidarité entre les peuples africains. La route panafricaine aurait la forme d'un triangle isocèle renversé, dont les trois angles seraient Le cap en Afrique du sud, Le Caire en Égypte et Dakar au Sénégal. La structure générale du réseau ferroviaire panafricain comprendrait un grand axe central allant du Caire au Cap, avec une variante AlgerLe Cap, une transversale Dakar-Mombassa, une boucle périphérique unissant toutes les zones côtières au cœur du continent, et plusieurs autres axes reliant entre elles les différentes régions. L'implantation de ces structures devrait se faire selon une nouvelle conception de l'intégration régionale ayant pour fondement le com-
\end{abstract}

* Chercheur au Centre d'études politiques (CEP), Faculté des Sciences sociales, administratives et politiques, Université de Kinshasa, RD Congo. E-mail : josebazonzi@yahoo.fr 
merce et l'investissement, l'exploitation concertée des ressources naturelles, l'implication du secteur privé, l'industrialisation axée sur les exportations ainsi que le développement des infrastructures efficaces et rentables, capables de briser le cercle de la pauvreté et les cycles de violences.

\section{Abstract}

Africa is a huge continent with so many landlocked regions. Therefore, there is need for adequate, efficient and highly varied transportation systems that can accelerate its development. The lack of adequate communication between production centres and densely populated areas with a high level of consumption is indeed the cause of a deep and pathological unbalance between supply and the global demand of goods and services throughout the continent. Besides the very costly air transport, it is desirable to invest in both road and railways transportations that are more accessible to the majority of the people and that are a genuine catalyst for Africa's integration and development. Thus, in order to contribute in the thinking on African development alternatives, we see it necessary to lay the foundations of a theoretical outline of a 'Pan African' network of roads and railways aimed at linking the continent's major economic and demographic areas in order to develop unity and solidarity between African peoples. The 'Pan African' road network would have the form of a reversed isosceles triangle, the three angles of which would be Cape Town in South Africa, Cairo in Egypt and Dakar in Senegal. The general structure of the Pan African railway network would include a big central route from Cairo to Cape Town with an Algiers-Cape Town variant, with a cross-country DakarMombassa line, a peripheral loop uniting all the continent's coastal areas, and many other highways linking different regions. Setting these structures should be done according to a new conception of regional integration based on trade and investment, a concerted tapping of natural resources, the implication of the private sector, export-based industrialization as well as the development of efficient and profitable infrastructures able to break the cycle of poverty and cycles of violence.

\section{Introduction}

Le transport est un secteur clé pour le développement d'un territoire donné. En effet, de tout temps et de surcroît dans une économie moderne, le transport joue un rôle prépondérant car il facilite non seulement les échanges entre agents économiques, mais également améliore la circulation des personnes et des biens, des idées et des services, ainsi que le raffermissement des liens d'amitié et de fraternité entre les peuples.

L'Afrique est un continent immense dont plusieurs contrées sont encore enclavées à ce jour. De ce fait, elle nécessite des systèmes de 
transport adéquats, efficaces et fort variés, car de l'intégration des différents systèmes de transport adaptés à son espace physique dépend en majeure partie son développement. En effet, l'ouverture et l'accroissement des échanges rendus possibles grâce auxdits systèmes ont pour rôle de stimuler la croissance économique.

Cependant, dans la plupart des pays africains, il existe un déficit de liaisons routières et/ou ferroviaires entre les grandes métropoles et l'arrière-pays, d'une part, et d'autre part, entre les différentes localités à l'intérieur du pays, et les différents États entre eux. Il s'agit donc de repenser et de réorganiser les liaisons entre les centres de production et ceux de consommation, et d'opérer le désenclavement des différentes zones de production et de peuplement.

Par ailleurs, il s'avère que le manque de communication adéquate entre la ville et la campagne d'un côté, et entre les zones de production et celles de forte densité et consommation de l'autre, se trouve à la base du développement déstructuré de l'espace africain, du fait d'un déséquilibre profond et pathologique entre l'offre et la demande globales des biens et services.

À titre de rappel, notons que de façon globale la structure des réseaux de transport que la plupart des pays africains ont hérité de la période coloniale n’a pas évolué du tout. Ces réseaux étaient conçus pour faciliter l'acheminement des produits agricoles et miniers vers la colonie. C'est ainsi que plusieurs pays éprouvent jusqu'à ce jour d'énormes difficultés pour l'organisation de leur marché interne, ainsi que la régulation des échanges avec leurs voisins.

En revanche, bien qu'il existe des liaisons aériennes entre les principales capitales africaines, il n'est pas superflu de rappeler que le transport aérien reste onéreux et n'est utilisé que par une infime partie de la population, ce mode de transport étant largement inaccessible à la majorité de la population africaine à cause notamment de son coût élevé. Dès lors, il s'avère nécessaire d'explorer d'autres modes de transport, plus modestes et plus accessibles à la majorité de la population africaine. Nous pensons donc aux modes traditionnels du transport routier et ferroviaire, qui s'affirment comme étant des catalyseurs du développement intégré et de l'aménagement de l'espace, et des moyens par excellence d'intégration des différentes régions du continent africain.

Ainsi, il nous semble nécessaire, alors même que la mondialisation devient non seulement globale et prégnante, mais également irréversi- 
ble et incontournable, de jeter les bases sur l'esquisse théorique d'un réseau routier et ferroviaire à l'échelle du continent.

La route panafricaine, dont le but serait de relier les grandes aires économiques et démographiques du continent, aurait la forme d'un triangle isocèle renversé, dont les trois angles seraient Le Cap en Afrique du Sud, Le Caire en Egypte, et Dakar au Sénégal (cf. figure I en annexe). Des routes secondaires, en forme de Y et incrustées dans le continent profond, seraient reliées au grand triangle, et le tout formant un joli polyèdre, à la structure d'un diamant (cf. figure 2 en annexe).

Quant aux chemins de fer, leur implantation doit correspondre non seulement au tracé de grands pôles de croissance dynamique de chaque région, en conformité avec les analyses des institutions financières crédibles telle la Banque Africaine de Développement (BAD), mais également aux grandes aires démographiques du continent. En plus, dans le contexte de la préservation de l'écosystème notamment la réduction de l'émission de gaz à effet de serre, le chemin de fer présente des atouts fort intéressants dans le cadre de l'aménagement du territoire et de la qualité de vie des populations sur le continent africain.

Mais, étant donné que le secteur des transports requiert de lourds investissements, et qu'il constitue sans conteste l'une des dix priorités du Nouveau partenariat pour le développement de l'Afrique (NEPAD), le pilotage de ce macroprojet et de tant d'autres qui s'ensuivront, dans le cadre des accords de coopération panafricaine, sollicite, à tous les niveaux, l'engagement des leaders africains, l'amélioration des capacités managériales des cadres africains, ainsi que l'implication effective des bailleurs de fonds et des investisseurs privés dans l'optique du partenariat public/privé.

Nous pouvons affirmer avec Ghebray Berhane (1992:35-40) ${ }^{1}$ que l'inefficacité des systèmes de transports africains (routiers, ferroviaires, fluviaux et maritimes) et même des services de télécommunications sont des réelles entraves aux divers échanges régionaux : ils alourdissent le coût de l'activité économique africaine. Ainsi, l'Afrique doit aspirer vers son développement intégral, en luttant contre la dispersion imposée par la distance, et en assurant la promotion du transport des biens, des services et des personnes. Aussi, doit-t-elle encourager la libéralisation multilatérale des échanges et l'intégration régionale effective, car elles sont source de gains dynamiques et d'élargissement du marché, qui à leur tour génèrent la croissance de la production et les économies 
d'échelle. Enfin, l'intégration économique peut accroître le rendement du capital dans les pays intégrés, et ce, indépendamment du stock de capital (Baldwin 1992: 132).

La présente communication cherche donc à approfondir la réflexion sur :

i) le rôle d'un système de transports intégré dans le développement du continent africain, en l'occurrence le rôle stratégique de la route et du chemin de fer comme catalyseurs de son développement,

ii) l'esquisse du réseau routier et ferroviaire panafricain et,

iii) la nouvelle conception de l'intégration régionale.

\section{Rôle stratégique de la route et du chemin de fer comme catalyseurs du développement du continent africain}

Les transports, toutes modalités confondues, sont des facteurs d'intégration physique et économique des pays, et des vecteurs du développement de la production, de la promotion des échanges et de la cohésion sociale. En effet, comme le souligne Michel Norro (1998 :31), le secteur économique moderne des pays africains a comme caractéristique structurelle principale d'être intégré à un espace géographique extérieur. Et il apparaît clairement que cette situation d'extraversion est une situation fondamentalement précaire.

\section{Les principales fonctions de la route et du chemin de fer}

La route et le chemin de fer sont destinés à remplir certaines fonctions traditionnelles dont les principales peuvent être identifiées comme étant celles de faciliter l'approvisionnement et l'acheminement des produits sur les marchés, de permettre la libre circulation des personnes, des biens, des services et des valeurs (commerce, culture, technologie, savoir, paix,...), de contribuer à l'accroissement des flux d'échanges physiques, commerciaux, financiers et de facteurs de production entre les États des différentes sous-régions afin de soutenir la réduction de la pauvreté et le développement socio-économique.

Toutefois, à côté de ces fonctions traditionnelles, la route et le chemin de fer panafricains sont appelés à jouer un rôle stratégique destiné à améliorer l'insertion du continent africain dans l'ordre économique mondial. 
Ce rôle stratégique consiste à :

- contribuer à l'accélération et à la concrétisation de l'intégration régiona-le et de l'unité africaine ;

- permettre le désenclavement de plusieurs États incrustés dans l'Afrique profonde ; ce qui aura pour conséquence le renforcement de la vigueur économique globale de la région Afrique ;

- contribuer à l'élargissement de la taille des marchés au sein et au-delà de la région ; ce qui va créer un environnement incitatif favorable à la promotion du secteur privé et à l'investissement ;

- améliorer l'aménagement du territoire et l'attraction des investissements directs étrangers (IDE) générateurs des emplois substantiels dans la région ;

- encourager l'engagement et le déploiement sur le marché africain des investissements directs des nationaux ( «IDN »), gage de l'attrait et de la consistance des investissements directs étrangers (IDE) ;

- faciliter l'accès des populations environnantes aux différents marchés et services sociaux induits par la présence de la route et du chemin de fer (petit commerce, écoles, hôpitaux, etc.) ; ce qui conduit par effet d'entraînement à la réduction de la pauvreté dans les zones concernées ;

- aider à la promotion de l'émancipation des femmes et des groupes défavorisés au sein des communautés (éclosion des activités agro-pastorales, du petit commerce et de l'artisanat caractéristiques des économies populaires africaines, ...).

Mais, appréhendées de façon globale, les deux modalités de transport, à savoir la route et la voie ferrée, ont comme par vocation, une double mission à accomplir : la mission de circulation et celle de développement. Nous allons dire un petit mot sur cette dernière.

\section{La mission externe de la route et du chemin de fer}

La route et le chemin de fer, comme nous l'avons déjà dit, sont deux modes de transport par excellence qui favorisent l'aménagement d'un territoire donné ainsi que son développement. Plusieurs études permettent d'apprécier cette affirmation à sa juste valeur; mais dans le cadre de cette communication, nous n'en retiendrons qu'une seule qui nous semble pertinente (Kyriacos 1984: 365-370).

En effet, Kyriacos pense que la route a une double mission, à savoir une mission interne ou de circulation qui est de satisfaire une demande 
de trafic entre deux points, et une mission externe ou de développement qui est de satisfaire des objectifs d'aménagement de territoire et de développement. En fait, suite au développement rapide de l'industrie de l'automobile, la route a acquis une place prépondérante dans les infrastructures de transport, tandis que l'économie des transports a été plus centrée sur la recherche de l'optimum interne sur des liaisons isolées, dans le but de parer à un besoin spécifique de transport. Or cette vision qui ne correspond qu'à la première mission de la route, à savoir la mission de circulation, est souvent la cause d'échec de nombreux programmes routiers. Car les rapports de la route avec son environnement sont plutôt dynamiques et relèvent d'un autre aspect beaucoup plus global, la mission de développement. Ainsi, il est nécessaire de considérer les rapports de la route avec son environnement sous deux angles, l'organisation de l'espace et le développement de la région irriguée.

Concernant l'organisation de l'espace, la théorie de la polarisation de l'espace montre que la vie économique et sociale est non seulement faite d'échanges mais également qu'elle ne se manifeste pas partout de façon homogène. Elle s'organise autour des centres ou « pôles » d'intérêt qui concentrent entre eux une floculation de peuplement et d'activités avec des intensités d'attractions mutuelles variables, et les flux qui en résultent peuvent être mis en évidence par les résultats d'enquêtes. Il s'établit donc, dans un certain rayon d'influence entre un pôle d'intérêt et des agglomérations satellites (villes) ou les localités (campagnes) qui gravitent autour, un courant d'échanges réciproques dont l'intensité interne est supérieure en chaque point à l'intensité externe. Et comme les pôles d'intérêt n'ont pas tous la même importance, on peut les « hiérarchiser » en pôles primaires, secondaires, tertiaires, quaternaires, ... suivant que leur influence s'exerce à l'échelle continentale (régionale), sous-régionale, nationale, provinciale, etc.

En ce qui concerne les effets structurants du réseau routier sur l'environnement, il semble que l'effet multiplicateur de l'investissement et l'effet accélérateur de l'investissement sont les plus plausibles, lorsque la mission externe du réseau routier est bien remplie. En agissant sur la diminution des coûts des moyens de production, le réseau de transport, par sa création et surtout par sa qualité, est l'un des facteurs-clés pouvant induire l'essor d'une contrée ou d'un territoire donné. Il est dès lors important, par souci d'équilibre, d'apporter à la campagne l'infrastructure routière adéquate couplée à l'investissement pour contribuer à la fixation de la population paysanne. Car la concentration des infras- 
tructures de transport en un pôle géant a pour effet de «vider » la population des campagnes environnantes au profit de la mégapole. C'est le cas de Beyrouth qui est devenue une "métropole asphyxiée » (Kyriacos 1984: 367).

Quant à la relation entre la route et le développement de la zone irriguée, il n'y a pas de façon formelle un lien de cause à effet entre les deux; cependant, il n'est pas moins vrai qu'à côté du dynamisme et de l'esprit d'entreprise des riverains, la route est l'un des nombreux facteurs dont la combinaison concourt audit processus, et à ce jour, son rôle de catalyseur n'est plus à démontrer. En fait, « le développement suppose un environnement favorable, une combinaison de circonstances mais également une combinaison d'actions concertées dans différents secteurs, qui pose à son tour des problèmes de synchronisation dynamique et fonctionnelle» (Kyriacos 1984 : 369). En définitive, le développement résulte d'une action concertée et coordonnée nécessitant la contribution prépondérante de la route.

\section{Esquisse théorique du réseau routier et ferroviaire panairicain}

Ce réseau, nous le qualifions de «panafricain », car il est appelé à développer l'unité et la solidarité entre les peuples africains. Actuellement, l'opportunité exceptionnelle qu'offre le Nouveau Partenariat pour le Développement de l'Afrique (NEPAD) nous autorise de réfléchir profondément sur l'aménagement de l'espace africain en vue de favoriser non seulement la valorisation de son énorme potentiel physique et humain, et la réduction des distances entre les différents pays africains mais également l'accroissement et l'intensification des échanges intrarégionaux, et partant une amélioration significative des relations panafricaines.

La route et la voie ferrée panafricaines ont pour vocation de relier autour d'un réseau primaire les principales villes des différents pôles économiques et démographiques du continent. Car, de plus en plus, les villes ont la capacité d'agir sur l'information concernant les marchés internationaux, la flexibilité des structures commerciales et productives, et la capacité d'accéder à des réseaux de dimensions et complexités variées (Borja 1996 ; Simone 1998 : 28).

En outre, comme l'écrit si bien AbdouMaliq Simone (1998:29) :

Les villes viables font office de centres régionaux, de portes d'entrée, de réseaux de distribution, de spécialistes sectoriels, de centres d'activités 
des transports, ou alternativement, de sites de production où les coûts sociaux restent fiables, où une main-d'œuvre bon marché est mobilisée, et qui amènent toutes sortes d'arrière-pays dans les circuits de production régionaux et mondiaux.

\section{Le réseau routier}

Les routes principales et secondaires africaines représentent moins de $50 \%$ de l'ensemble du réseau routier $(1500000 \mathrm{~km})$ dont la densité $\left(0,05 \mathrm{~km}\right.$ par $\left.\mathrm{km}^{2}\right)$ est la plus faible du monde (Atlas de l'Afrique 2000 :50). De plus, cette infrastructure routière est insuffisante : seuls $12 \%$ des routes ont un revêtement dur (Muhigirwa 2003 : 23).

$\mathrm{Au}$ plan théorique, un réseau routier assure une desserte spatiale satisfaisante aux conditions suivantes :

- l'espace doit être parcouru par les différentes classes de routes (primaires, secondaires, tertiaires, ...) afin d'éviter tout déséquilibre et tout enclavement ;

- chaque route doit fonctionner selon sa classe ou sa catégorie sans entraves ;

- le réseau routier et le réseau de polarisation doivent être harmonieusement répartis afin de permettre à la vie économique et sociale de se développer d'une manière équilibrée aux trois niveaux : régional ou continental, sous-régional ou zonal - les différentes zones ou sous-régions étant le Nord, le Sud, le Centre, l'Ouest et l'Est) - et national ou local (au niveau de chaque pays).

Il faut en outre aborder le problème de l'aménagement en tenant compte de la rentabilité en fonction de la demande de trafic, en termes de structure, de conditions de relief et de géométrie. En effet, la rationalité économique doit rester au cœur de ce macroprojet pour lui donner toutes les chances de réussite et d'essor économique. Car dans le passé, l'Afrique a connu plusieurs projets similaires qui se sont soldés en échec cuisant à cause de l'absence de cette dimension. Autant la volonté politique montre ses limites sans rationalité économique et l'implication communautaire, autant la rationalité économique est obsolète sans retombée sociale positive et palpable.

Étant donné la polarisation de l'espace physique et la structure polarisée des échanges, le réseau routier que nous proposons (cf. figure 3 en annexe) sera composé d'une grande boucle centrale en forme de triangle isocèle renversé et d'une boucle périphérique (réseau primaire qui 
relie les pôles primaires du continent ou de la région Afrique), et de plusieurs sous-ensembles. Ceux-ci comprennent des triangles multiformes structurés en réseau secondaire composé des pôles secondaires mettant en liaison les différentes sous-régions. Le réseau tertiaire, quant à lui, est formé de petits triangles dont les principaux axes routiers sont également en forme de $\mathrm{Y}$ : ce sont les pôles tertiaires qui ont pour rôle de relier les différents pays d'une sous-région. Enfin, le réseau quaternaire est constitué des tronçons routiers, qui sont en fait des mailles du réseau tertiaire et qui connectent les villes aux localités secondaires au niveau des espaces nationaux : les pôles quaternaires de ce réseau ont la vocation de désenclaver les campagnes africaines.

Ainsi, le réseau routier panafricain proposé est dense et comprend quatre principaux niveaux de réseau. Il s'agit du réseau primaire, avec ses deux boucles, centrale et périphérique, du réseau secondaire avec des axes interrégionaux, du réseau tertiaire avec des tronçons interétatiques, et du réseau quaternaire avec des maillons intranationaux.

De manière concrète, la grande boucle centrale relie la ville du Cap à celle de Dakar et du Caire, et met en liaison les différentes villes et localités suivantes : Le Cap (Afrique du Sud), Tshabong, Ghanzi (Botswana), Andara (Namibie), Sesheke, Mongu (Zambie), LumbalaKaquengue, Luau (Angola), Dilolo, Mbuji-Mayi, Lubefu, Kisangani, Buta, Mobaye (RDC), Bambari, Birao (RCA), Wau, El-Obeïd, Khartoum, Atbara, Ouadi-Halfa (Soudan), Abou-Simbel, Assouan, Louxor, Le Caire (Égypte), Awila, Mourzouk, Ghât (Libye), Tamanrasset (Algérie), Tombouctou (Mali), Dakar (Sénégal), Kayes, Bamako (Mali), Ouagadougou (Burkina Faso), Abuja (Nigeria), Yaoundé, Ebolowa (Cameroun), Koulamoutou, Franceville (Gabon), Oyo, Brazzaville (Congo), Kinshasa, Matadi (RDC), Luanda, Lobito, Namibe (Angola), Tsumeb, Windhoek (Namibie) et Le Cap (Afrique du Sud).

Le réseau secondaire comprend principalement les axes routiers qui relient entre elles les différentes sous-régions. Tel est l'exemple de l'axe routier Yaoundé (Cameroun) - Abuja - Kano (Nigeria) - Agadez - Arlit (Niger) - Tamanrasset - In Salah - Alger (Algérie). Cet axe a l'avantage d'opérer une distribution fonctionnelle efficiente des routes au niveau du réseau secondaire : il occupe une position médiane dans la région et exerce à merveille sa fonction sous-régionale. En effet, il permet de relier la zone du Nord à la zone de l'Ouest et à celle du Centre, et il est connecté au réseau primaire qui relie Dakar au Caire et au Cap. 
Plusieurs autres axes prévus dans l'esquisse théorique ont la prétention de jouer ce rôle. On peut donc citer à titre illustratif l'axe Bangui (RCA) - Lisala - Kisangani - Isiro (RDC) - Kampala (Ouganda) - Juba - Malakal - El-Obeïd - Khartoum - Port-Soudan (Soudan) - Hourghada - Le Caire (Égypte). Cet axe routier permet la jonction des zones africaines du Centre, de l'Est, du Nord et de l'Ouest dans le prolongement Bangui-Yaoundé, Douala et Lagos, et du Sud dans l'axe central (prolongement Kisangani - Mbuji-Mayi - Dilolo - Luau - Mongu - Ghanzi Tshabong - Le Cap), car il est lui aussi incrusté au réseau primaire qui va du Caire au Cap et à Dakar.

L'examen de la figure 3 nous fait également découvrir quelques tronçons routiers en forme de $\mathrm{Y}$ destinés à désenclaver l'Afrique profonde. En voici quelques exemples.

Pour la zone du nord : Tamanrasset, In Salah (Algérie), Rabat (Maroc), via Bechar, Alger (Algérie), Ghât, Ghadamès (Libye), Tunis (Tunisie), Tripoli (Libye).

Pour la zone de l'ouest: Abidjan (Côte d'Ivoire), Bobo-Dioulasso (Burkina Faso), via Yamoussoukro (Côte d'Ivoire), Bamako (Mali), Ouagadougou (Burkina Faso), Porto Novo (Bénin), Niamey (Niger), Tombouctou (Mali), via Gao (Mali), Agadez (Niger).

Pour la zone de l'est: Kampala (Ouganda), Juba, Wau (Soudan), Addis-Abeba (Ethiopie).

Pour la zone du sud : East London (Afrique du Sud), Maseru (Lesotho), Johannesburg, Pretoria (Afrique du Sud), Mbabane (Swaziland), Maputo (Mozambique).

Pour la zone du centre : Brazzaville (Congo), Franceville (Gabon), Yaoundé (Cameroun), Bangui (RCA), Kisangani (RDC), Bangui (RCA), Yaoundé (Cameroun), N'Djamena (Tchad).

Le réseau routier proposé ci-dessus permet d'obtenir une desserte acceptable et un désenclavement de la plupart des contrées incrustées dans le continent.

\section{Le réseau ferroviaire}

Lactuel réseau ferroviaire africain est le moins dense du monde : il totalise environ $85000 \mathrm{~km}$, soit une densité de 2,8 km de voies pour I $000 \mathrm{~km}^{2}$ (contre 60 en Europe, par exemple) (Atlas de l'Afrique 2000 : 50). Et ce réseau est caractérisé, à l'exception de quelques lignes près, par l'existence de courts tronçons qui vont des zones minières ou de 
production de cultures d'exportation vers les régions côtières, accentuant ainsi la situation d'extraversion et donc de précarité de l'économie africaine évoquée plus haut.

Pour pallier à cette anomalie, le réseau ferroviaire proposé à la figure 4 en annexe voudrait atteindre deux buts majeurs, à savoir établir une liaison entre les principales zones de peuplement de toutes les sousrégions d'Afrique, afin de permettre une meilleure circulation des personnes et des marchandises, ainsi que relier ces zones aux différents pôles de croissance économique à l'intérieur du continent.

En effet, cinq pays représentant près de la moitié (environ $43 \%$ ) de la population africaine, hormis quelques autres pays, sont traversés par l'axe ferroviaire central et sa variante. Il s'agit du Nigeria (Afrique de l'Ouest), de l'Égypte (Afrique du Nord), de l'Ethiopie (Afrique de l'Est), de la RDC (Afrique centrale), et de l'Afrique du Sud (Afrique australe).

Ainsi, la structure générale de ce réseau comprend un grand axe central allant du Caire au Cap, avec une variante Alger-Le Cap, une boucle périphérique qui unit toutes les zones côtières au cœur du continent, et plusieurs autres axes qui relient entre elles les différentes sous-régions.

Les principaux nœuds ferroviaires de l'axe central (épine dorsale) sont : Le Caire, Louxor, Abou-simbel (Égypte), Ouadi-Halfa, Khartoum, El-Obeïd (Soudan), Addis-Abeba (Éthiopie), Juba (Soudan), Kisangani, Kindu, Lodja, Mbuji-Mayi, Mwene-Ditu, Lubumbashi (RDC), Lusaka (Zambie), Bulawayo (Zimbabwe), Gaborone (Botswana), Johannesburg, Kimberley, Le Cap (Afrique du Sud).

La variante va d'Alger au Cap en passant par In Salah, Tamanrasset (Algérie), Arlit, Agadez (Niger), Kano (Nigeria), N'djamena, Sarh (Tchad), Bangui (RCA), Yaoundé (Cameroun), Franceville (Gabon), Oyo, Brazzaville (Congo), Kinshasa, Matadi (RDC), Luanda, Huambo (Angola), Windhoek (Namibie), Le Cap (Afrique du Sud).

Comme on peut bien le voir dans la figure 4 en annexe, l'axe ferroviaire central relie le nord, l'est, le centre et le sud du continent, tandis que sa variante permet la connexion des parties septentrionale, occidentale, centrale et australe.

Quelques axes latéraux ainsi que leurs variantes sont également prévus dans cette esquisse. Ainsi en est-il de la voie ferrée devant relier Dakar au port de Mombasa et qui traverse Kayes, Bamako, Bobo- 
Dioulasso, Ouagadougou, Niamey, Kano, N’Djamena, Sarh, Bangui, Bumba, Kisangani, Bunia, Kampala et Naïrobi.

Les axes Tripoli-Dakar, Tripoli-Abidjan, Alger-Lagos, Dakar-Djibouti, Douala-Dar es-Salaam, Banana-Maputo, Luanda-Durban, etc. trouvent leur place dans ce vaste réseau à vocation panafricaine.

En définitive, l'on peut affirmer que la complémentarité route / rail au sein du réseau routier et ferroviaire panafricain a pour principal effet de raccourcir la distance entre différents points de l'espace africain, d'éviter la rupture des charges-en ce qui concerne le transport des marchandises-et d'encourager l'amitié entre les peuples et l'accroissement des échanges intrarégionaux. Et ceci est rendu possible grâce à l'action concrète d'une volonté politique éprouvée et traduite en acte par le biais d'une intégration régionale effective fondée sur une approche nouvelle.

\section{Nouvelle conception de l'intégration régionale}

La plupart des scientifiques, politiques et acteurs de développement sont d'avis que le processus d'intégration en Afrique piétine depuis plus d'une décennie et qu'il est aujourd'hui nécessaire de lui donner une nouvelle envergure.

En effet, dans une étude récente sur l'intégration en Afrique, Cadot et al. (2005) estiment qu'il est urgent de développer de nouvelles approches à la politique commerciale des pays d'Afrique subsaharienne. Ces auteurs invoquent pour cela deux raisons essentielles : d'une part, les précédentes tentatives d'intégration régionale ont dans une large mesure échoué par rapport à leurs objectifs ambitieux, et d'autre part, la faible performance des pays africains est sans conteste due, au moins en partie, à leurs faibles résultats commerciaux.

D'ailleurs, les accords régionaux de libre échange sont susceptibles d'essuyer un échec parce que la plupart des pays africains ont des structures d'échanges très semblables, important et exportant des biens similaires. Ce qui dénote fort bien une structure des échanges très substituable. En outre, l'analyse standard de Viner sur les accords commerciaux préférentiels (Cadot et al. 2005) passés dans le cadre d'une zone de libre échange montre que le potentiel de création de commerce pour cette zone-qui accueillerait des accords de libre échange (ALE) régionaux-est faible. 
Nous estimons pour notre part que la nouvelle conception de l'intégration régionale doit être basée sur une approche qui consacre non seulement l'ouverture accrue au commerce et à l'investissement, qui permet des économies d'échelle et favorise la concurrence, la réalisation de projets communs d'exploitation de ressources naturelles et de production de biens, l'inter-connexion des pays africains par des services d'infrastructures efficaces (Kabbaj 2000), mais également l'encouragement et l'engagement du secteur privé qui doit devenir le principal support de la croissance économique régionale, l'industrialisation axée sur les exportations et le développement des infrastructures communes de transport et de télécommunications.

\section{Le commerce et l'investissement}

En tant que créateur d'emplois et de richesses, le commerce est un moteur vital de la croissance économique. L'exemple de la Chine est éloquent : sa croissance fulgurante procède d'une augmentation forte et continue de la productivité (doublée d'une efficacité accrue des travailleurs) rendue possible grâce à des réformes axées sur le marché. Le commerce permet également d'atténuer les conflits tribalo-ethniques et les visées expansionnistes des communautés. Ce qui constitue un effet favorable à la paix sociale et à l'investissement. Le commerce est en effet très ancien, et à ce sujet, Montesquieu disait : «L'effet naturel du commerce est de porter à la paix. Deux nations qui négocient ensemble se rendent réciproquement dépendantes : si l'une a intérêt d'acheter, l'autre a intérêt de vendre sur des besoins mutuels » (Bakandeja 2001 : 9).

\section{l'exploitation concertée des ressources naturelles}

Au niveau infrarégional et même régional, plusieurs initiatives d'exploitation des ressources à fortes économies d'échelle sont un facteur catalyseur de l'intégration. Ainsi en est-il du projet de rentabilisation du potentiel hydro-électrique du site d'Inga en République Démocratique du Congo (RDC), susceptible de jouer un rôle déterminant dans la production de l'énergie ainsi que sa fourniture dans une bonne partie du continent aux fins de soutenir la localisation optimale des industries. Les premiers bénéficiaires de cette option de développement devraient vraisemblablement être les pays de l'Afrique australe regroupés au sein de la $\mathrm{SADC}^{2}$, ceux de l'Afrique centrale regroupés au sein de la 
$\mathrm{CEMAC}^{3}$ et de la CEPGL ${ }^{4}$, ainsi que ceux de l'Afrique orientale organisés autour de la COMESA ${ }^{5}$.

En fait, une des causes d'échec de l'intégration régionale dans le passé était l'incapacité des dirigeants africains à aboutir à un accord sur la localisation stratégique des investissements industriels. Ainsi, il faut considérer l'intégration comme un véritable moyen de promotion de l'investissement in loco et une méthode de captation des flux de capitaux destinés à nourrir tous les secteurs de l'économie.

\section{Développer des inirastructures eificaces et briser le cercle de la pauvreté}

Ensuite, il appert que la faiblesse des infrastructures est un obstacle majeur au commerce et au développement durable dans les pays africains. C'est pourquoi le développement des infrastructures constitue un élément-clé dans la stratégie de réduction de la pauvreté, loin s'en faut. Et tandis qu'au niveau macro-économique, les investissements dans les infrastructures sont intimement liés à la croissance économique et à l'intégration dans l'économie mondiale, au niveau microéconomique, l'accès auxdites infrastructures permet aux ménages de sortir du cercle vicieux de la pauvreté et d'avoir accès aux marchés.

\section{L’implication du secteur privé}

Par ailleurs, l'Afrique offre souvent aux yeux du monde un spectacle désolant, celui d'une terre de famine, de guerres, de maladies et de conflits divers. Avec ses 800 millions de consommateurs dont près de la moitié se contentent quotidiennement de moins de 1 dollar américain pour vivre, elle éprouve d'énormes difficultés pour accélérer la réduction de la pauvreté, étant donné les taux de croissance actuels qui sont largement insuffisants. Or l'accélération de la croissance économique passe notamment par la promotion du secteur privé, le renforcement des ressources humaines, l'amélioration des réformes économiques et institutionnelles, l'utilisation rationnelle des ressources naturelles et la promotion d'une gouvernance de qualité et de l'intégration régionale efficace. En effet, les réformes institutionnelles sont nécessaires pour la transparence des marchés, et le modèle libéral de l'économie de marché est basé sur les valeurs telles la liberté, la démocratie, l'État de droit et la bonne gouvernance (Igué 1999 : 56), valeurs que l'Afrique tarde à s'approprier. 


\section{Une industrialisation axée sur les exportations}

Enfin, l'industrialisation axée sur les exportations doit privilégier l'industrie manufacturière car elle est un facteur diversificateur de l'économie et créateur d'emploi. Elle est considérée comme un atout beaucoup plus sûr pour le développement économique de l'Afrique (Hawkins 1990 :270). Qui plus est, le secteur industriel génère la valeur ajoutée la plus forte et la plus fiable dans le développement autocentré d'un pays (Tshibangu $2004: 118$ ). Et il ne nous semble pas inutile de rappeler que l'industrie est un élément fondamental en ce qui concerne la réorganisation de la structure de production. Plus particulièrement, « l'industrialisation contribue au développement en aidant à la transformation du comportement individuel et de l'organisation sociale » (Norro 1998 :172). En outre, l'industrie manufacturière utilise les technologies, crée des compétences et des connaissances, encourage l'esprit d'entreprise et l'innovation, favorise la mobilité sociale, génère de nombreux emplois et revenus et constitue un pôle d'attraction pour les investissements étrangers (BAD 2002 :26), lesquels investissements sont indispensables pour le développement accéléré et harmonieux des infrastructures communes de transport et des télécommunications.

\section{Surmonter les principaux défis de l'intégration}

Mais, tout ce qui précède, c'est-à-dire le pilier de la nouvelle conception de l'intégration, nécessite la mise en place des mécanismes concertés pour l'harmonisation des politiques macroéconomiques et sectorielles, la réalisation des macroprojets visant à valoriser les ressources naturelles et humaines des différents partenaires en vue de la production commune des richesses, la création des systèmes de paiements et de chambres de compensation en attendant le processus ultime d'unification monétaire, et bien sûr «l'interconnexion des pays africains par des services d'infrastructures efficaces ", à l'instar du réseau intégré de routes et de chemins de fer panafricain décrit ci-dessus.

Car, faut-il le rappeler, par manque de réseaux d'infrastructures et de transports, les économies africaines demeurent peu articulées; elles sont constituées en sous-systèmes peu communicants au niveau des flux, des prix et des informations (Hugon 2001 : 38).

C'est pourquoi, pour valoriser son énorme potentiel physique et humain, l'Afrique doit avancer résolument sur la voie de l'intégration 
régionale selon cette nouvelle approche et hâter ainsi l'application du Traité d'Abuja dont les principaux thèmes s'articulent autour des concepts de solidarité et d'autonomie collective, d'une stratégie de développement autonome et endogène, et d'une politique d'autosuffisance pour la satisfaction des besoins alimentaires (Banque africaine de développement (BAD), $2000:$ 125).

Car en effet, en dépit des progrès fort louables réalisés par quelques États africains, les différents mécanismes d'intégration régionale n’ont pas encore réussi à accroître le volume total des échanges entre pays africains, améliorer la croissance économique globale du continent ou développer le commerce intra-africain, à cause notamment de «l'absence de réelle volonté politique » (Norro 1998:201) et du manque d'un réseau d'infrastructures de communication intégré et efficace. Et des efforts supplémentaires doivent être réalisés dans le domaine de la «souveraineté » des États africains et de leur capacité à négocier et à juguler les effets pervers du commerce extérieur, de la confiance mutuelle entre partenaires, et de la valorisation de grandes langues africaines à vocation internationale ${ }^{6}$.

\section{Les bénéfices de l'intégration régionale}

Lintégration régionale dont l'objectif majeur est de créer un marché commun africain (avec libéralisation des mouvements de facteurs de production), comporte plusieurs avantages et est d'une importance primordiale pour le développement durable de l'Afrique. Elle engendre des gains dynamiques induits par les échanges, et la création des flux d'échanges contribue à l'élargissement du marché qui peut générer des économies d'échelle susceptibles d'attirer et de fixer durablement des investissements directs étrangers (IDE) dans la région.

Elle peut également, à travers des accords d'intégration régionale (AIR), mieux que les institutions nationales, promouvoir la crédibilité des politiques communes menées au sein des organisations supranationales, contribuer à prévenir les conflits entre différents pays, stimuler l'investissement et favoriser la localisation optimale des industries. La rentabilisation du site d'Inga évoquée ci-dessus et tributaire d'une demande croissante, en est un exemple éloquent et rentre bien dans la vision de cette nouvelle approche. 
L'Afrique a donc besoin d'initiatives d'intégration régionale qui visent une réduction sensible de coûts de transactions. Car, on le sait, basés sur les concepts de pôle de croissance et de retombées régionales positives, les avantages potentiels de l'initiative d'intégration régionale risquent d'être limités par la médiocrité des infrastructures de transport et de communication. C'est pourquoi la nouvelle approche de l'intégration régionale en Afrique doit désormais privilégier la création d'un réseau d'infrastructures efficace et intégré en vue de maximiser les avantages reconnus à une région intégrée bâtie autour de pôles de croissance dynamique. Nous en voulons pour preuve les résultats positifs enregistrés ces cinq dernières années dans la partie australe du continent.

\section{Une intégration qui se veut holistique}

La nouvelle approche dont il est question consacre l'intégration et le développement des capacités de production et des infrastructures locales. En fait, cette intégration se veut holistique en ce sens qu'elle comporte plusieurs dimensions (horizontale, verticale et physique) et a lieu à plusieurs niveaux (économique, politique et socioculturelle).

Lintégration horizontale fait référence à l'intégration des différentes sous-régions au sein du continent africain, tandis que l'intégration verticale rattache l'Afrique à la planète. La route et le chemin de fer «panafricains » constituent le fer de lance pour l'accélération de l'intégration physique du continent africain.

Au plan économique, la régionalisation doit être perçue comme un catalyseur pour intégrer l'Afrique dans l'économie mondiale (Kabbaj 2000). Au plan politique, la régionalisation doit permettre au continent de s'insérer dans la société politique internationale. Quant à l'intégration socioculturelle enfin, elle devra permettre d'unir les peuples africains autour de nouvelles valeurs socioculturelles et leur insertion à l'universel.

Ainsi, la nouvelle approche de l'intégration régionale doit contribuer à bâtir une nouvelle société africaine fondée sur une vision du développement durable qui procède de la rationalité économique, qui favorise l'unité et la solidarité entre les peuples ${ }^{77}$ Pourquoi pas un passeport africain pour tous les fils et filles du continent mère (« citoyens africains ») d'ici l'an 2020 pour couronner le processus d'intégration !et qui est capable d'accélérer l'insertion de l'Afrique dans le processus irréversible et prégnant de la mondialisation. 


\section{Conclusion}

Il est apparu, dès les indépendances, que la «balkanisation » de l'Afrique constituait un facteur de vulnérabilité extérieure, qu'elle limitait les possibilités de croissance interne et qu'elle réduisait son poids dans les négociations internationales (Hugon 2001 : 93). Cette assertion met en lumière la grande faiblesse qu'accusent encore de nos jours les infrastructures continentales, notamment en matière de transports et de communications.

En effet, jusqu'à ce jour, l'Afrique, pourtant dotée d'un espace immense au potentiel riche et diversifié, est encore caractérisée par un déficit routier et ferroviaire, déficit qui entrave les échanges et le développement du commerce intraafricain.

C'est pourquoi, dans le but de contribuer à la réflexion sur les alternatives au développement de l'Afrique, nous avons proposé dans cette communication, la construction imminente d'un réseau routier et ferroviaire intégré à l'ensemble de l'espace continental, fondée sur une nouvelle vision de l'intégration régionale. En effet, nous pensons que la route et le chemin de fer constituent sans conteste un élément essentiel pour l'aménagement de l'espace africain et un catalyseur de son développement intégré.

La route panafricaine en question est composée d'une grande boucle centrale en forme d'un triangle isocèle renversé qui relie les villes de Dakar, du Cap et du Caire, et d'une boucle périphérique qui relie toutes les villes et localités côtières du continent. Â l'intérieur du continent se dressent plusieurs axes routiers en forme de $\mathrm{Y}$ destinés à relier entre eux, les différents sous-régions et pays, et à désenclaver les contrées intérieures.

Le réseau ferroviaire, quant à lui, comprend, hormis la boucle périphérique et les axes secondaires, deux grandes structures : le réseau principal composé d'un axe ferroviaire principal allant du Caire au Cap avec une variante Alger-Le Cap, et un axe transversal reliant Dakar à Mombasa.

En outre ce réseau routier et ferroviaire panafricain a pour spécificité de relier les grandes aires démographiques aux grands pôles de croissance économique du continent, de manière équilibrée et intégrée, de façon à favoriser les échanges commerciaux et culturels et la rencontre des peuples. 
Cependant, il faut reconnaître que ces infrastructures si vitales pour le développement de l'Afrique, ne peuvent se réaliser et se concrétiser que dans le cadre d'une nouvelle vision de l'intégration régionale. Cette nouvelle approche met en effet un accent particulier sur le développement des infrastructures, en l'occurrence le réseau intégré de routes et de chemins de fer panafricain évoqué dans cette communication, et sur le rôle de l'investissement privé et du commerce intraafricain.

Aussi, peut-on affirmer sans détours avec Stephen Lewis (1990 : 475) que :

La véritable accession à une plus grande indépendance économique ne pourra s'effectuer qu'en appliquant des politiques qui conduisent à une exploitation plus efficace et plus effective des ressources du continent : la terre, le capital, les hommes, les devises, l'eau. Ces programmes doivent non seulement rentabiliser les possibilités qu'offre le commerce international, mais aussi limiter les effets pervers des marchés extérieurs sur les économies nationales.

En définitive, nous concluons avec Schulders (1991 : 26-27) :

En fin de compte, l'avenir des nations du Sud dépend des nations du Sud. Elles doivent convaincre le reste du monde (...) de leur aptitude à s'ouvrir sans perdre de vue les intérêts de leurs populations, à s'intégrer dans des ensembles cohérents sans ignorer ni bafouer les particularismes nationaux. (...) Car notre conviction est que l'Afrique du XXIe siècle sera une Afrique régionalisée et intégrée, ou elle ne sera plus.

Comme qui dirait, l'unité dans la diversité culturelle n'est que force mutuelle et enrichissement perpétuel. De cela dépendent aussi la vitalité et le développement de l'Afrique.

\section{Notes}

1. Cf. Allocution de Ghebray Berhane, Secrétaire Général du Groupe des États ACP, in J.-J. Symoens (dir.), 1992, Symposium « Les transports et les communications en Afrique », Bruxelles :Académie Royale des Sciences d'Outre-Mer, pp. 35-40.

2. South African Development Community, communauté regroupant la plupart des pays de l'Afrique australe. La RDC, par sa position stratégique au cœur du continent, en fait également partie.

3. Communauté économique et monétaire de l'Afrique centrale.

4. Communauté économique des pays des Grands Lacs.

5. Marché commun des pays de l'Afrique orientale. 
6. On peut actuellement penser à des langues telles le swahili, le wolof, le yoruba, le lingala, le zulu, le berbère,...

7. Pourquoi pas un passeport africain pour tous les fils et filles du continent mère («citoyens africains») d'ici l'an 2020 pour couronner le processus d'intégration!

\section{Références}

Atlas de l'Afrique, 2000, 2e édition, Paris: Groupe Jeune Afrique et Éditions du Jaguar.

Bakandeja wa Mpungu, G., 2001, Le droit du commerce international. Les peurs justifiées de l'Afrique face à la mondialisation des marchés, Bruxelles : De Boeck Université, Kinshasa : Afrique Éditions.

Baldwin, R.A., 1992, 'Measurable Dynamic Gains for trade', Journal of Political Economy, 100 : 162-174.

Banque Africaine de Développement (BAD),2000, Rapport sur le développement en Afrique. Intégration Régionale en Afrique, Paris : Economica : 132.

Berg, R. J. et J. Seymour Whitaker, 1990, Stratégies pour un nouveau développement en Afrique, Paris : Economica.

Berge, J., Le chemin de fer transafricain de l'Algérie au Cap, édité en 1912, http:// www.raidsahara.free.fr/histoiredusahara/extrait-l-livre.htm, 29 août 2005, http:/ /www.raidsahara.free.fr/histoiredusahara/le_chemin_de_fer_transafricain.htm, 3 septembre 2005 .

Berhane, G., 1992, « Allocution », in J.-J. Symoens, éd., Symposium Les transports et les communications en Afrique, Bruxelles : Académie Royale des Sciences d'OutreMer (ARSOM) : 35-40.

Cadot, O., J. de Melo et M. Olarreaga, 2005, L'intégration régionale en Afrique : où en sommes-nous? Asymetric Regionalism in Sub-Saharan Africa: Where do we Stand?

http://www.hec.unil.ch/ocadot/PAPERS/ABCDEFR.doc , 16 septembre 2005.

Hawkins, A.M., 1990, «L'Afrique peut-elle s'industrialiser? », in R. J. Berg, et J. Seymour Whitaker (éd.), Stratégies pour un nouveau développement en Afrique, Paris : Economica : 265-291.

http://www.afdb.org/fr/projets/appraisal reports/adf_bd_wp_2004_154_f.pdf, 21 août 2005.

http://www.africa-union.org/CIAD/Rapport, 29 août 2005.

http://www.ceeac-eccas.erg/docs/treaty/annexel l fr.pdf, 14 août 2005.

http://www.nepad.org/2005/fr/contactus.php, 23 septembre 2005.

http://www.unctad.org/fr/docs/aconf191_122.fr.pdf, 15 septembre 2005.

http://www.un.org/french/ecosocdev/geninfo/afrec/voll 6n²/162 perlf. htm-22k, 14 septembre 2005.

http://www.users.skynet.be/sky34004/hist_7.html, 18 septembre 2005.

Hugon, P., 2001 , Économie de l'Afrique, 3e éd, Paris : Ed. Découverte, coll. Repères. Igué, J. O., 1999, Le Bénin et la mondialisation de l'économie. Les limites de l'intégrisme du marché, Paris : Karthala. 
Kabbaj, O., 2000, « Avant-Propos », in Banque Africaine de Développement (BAD), Rapport sur le développement en Afrique. Intégration Régionale en Afrique, Paris : Economica.

Kyriacos, R., 1984, « Mission externe ou de développement de la route. Esquisse d'une théorie », in Routes et développement, Comptes rendus du Colloque International organisé par l'École Nationale des Ponts et Chaussées et l'Institut des Sciences et des Techniques de l'Equipement et de l'Environnement pour le Développement, Paris : Presses de l'Ecole nationale des Ponts et Chaussées, Vol. 1 : 365-370.

Lewis, S. R., 1990, « Le commerce africain et l'économie mondiale », in R. J. Berg, et J. Seymour Whitaker (éd.), Stratégies pour un nouveau développement en Afrique, Paris : Economica : 455-480.

Muhigirwa, R.F., 2003, NEPAD : le Nouveau partenariat pour le développement de l'Afrique, Afrique d'Espérance, n 1, oct. 2002 -janv. 2003, XIe année : 23-24.

Norro, M., 1998, Économies africaines: Analyse économique de l'Afrique subsaharienne, Bruxelles : De Boeck, 2e éd., coll. Ouvertures économiques.

Schulders, G., 1991, Développement : stratégie de l'urgence, Paris : L'Harmattan.

Simone, A., 1998, Mutations urbaines en Afrique, Dakar: CODESRIA, Document de travail $3 / 97$.

Symoens, J.-J., éd., 1992, Symposium « Les transports et les communications en Afrique », Bruxelles : Académie royale des sciences d'Outre-Mer (ARSOM).

Tshibangu, K.M., 2004, « Les mécanismes de blocage de l'industrialisation du Congo indépendant », in Quarante ans d'indépendance : Mythes et réalités, t. III, Questions économiques, Lubumbashi : Presses universitaires de Lubumbashi : 115130. 
Bazonzi: Systèmes de transport pour un développement intégré de l'Afrique 129

Figure 1. Le réseau routier et ferroviaire panafricain sous forme d'un triangle isocèle renversé (structure théorique à géométrie variable)

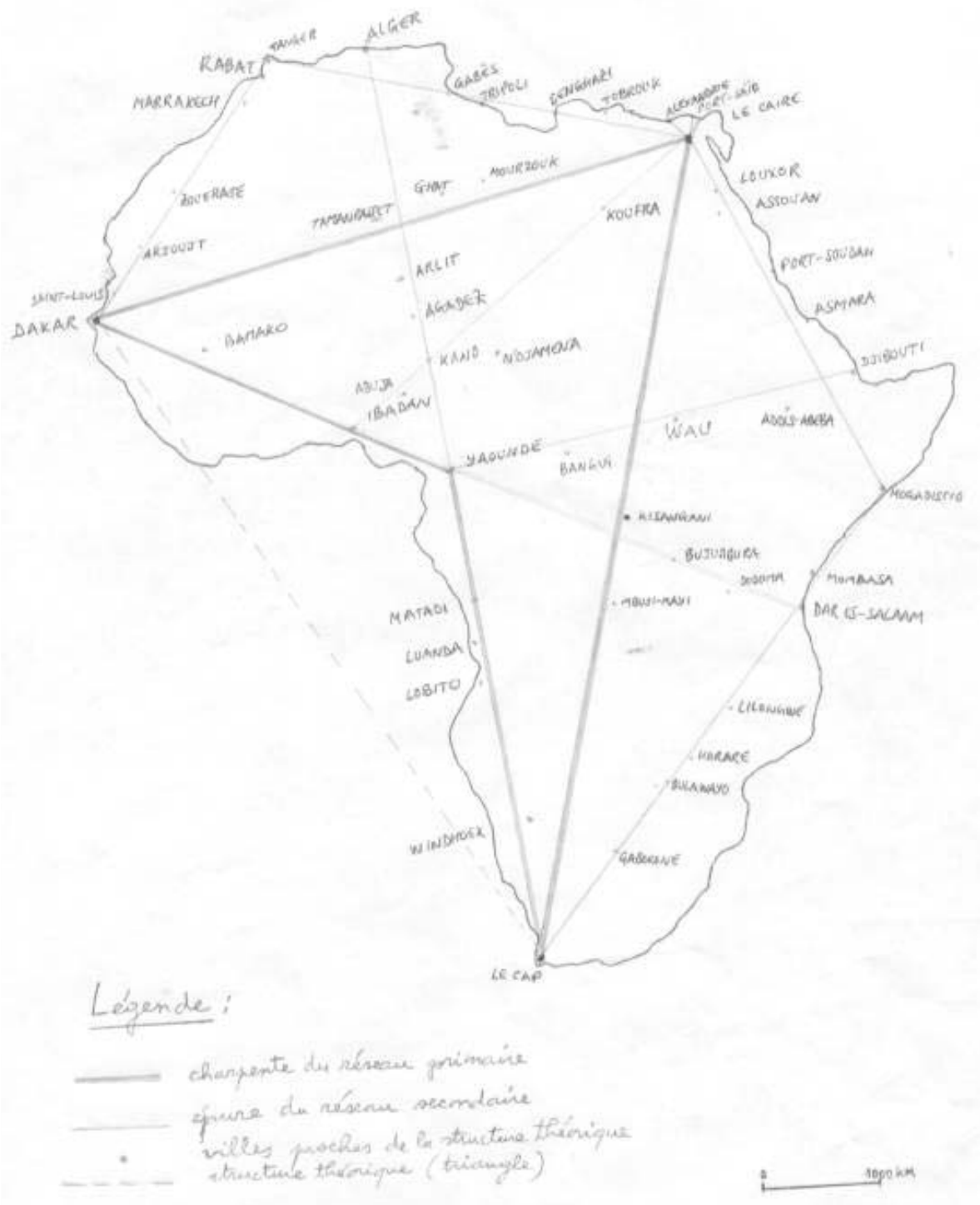


Figure 2. Le réseau routier et ferroviaire panafricain à la structure d'un diamant

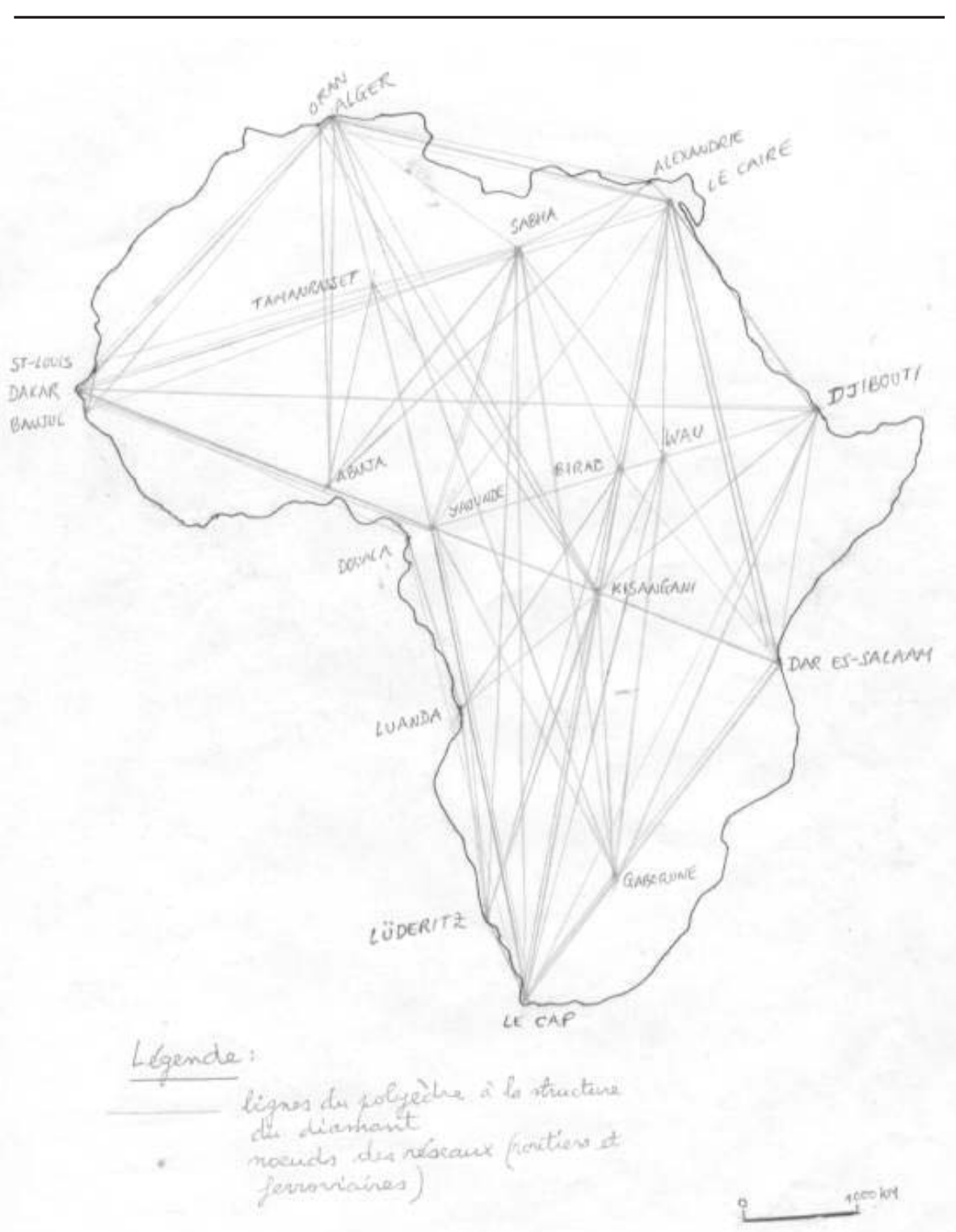


Bazonzi: Systèmes de transport pour un développement intégré de l'Afrique 131

Figure 3. La route panafricaine : structure théorique du réseau primaire et secondaire avec ses principaux nœuds et arcs




Figure 4. Le chemin de fer panafricain : structure théorique avec les principaux nœuds et arcs

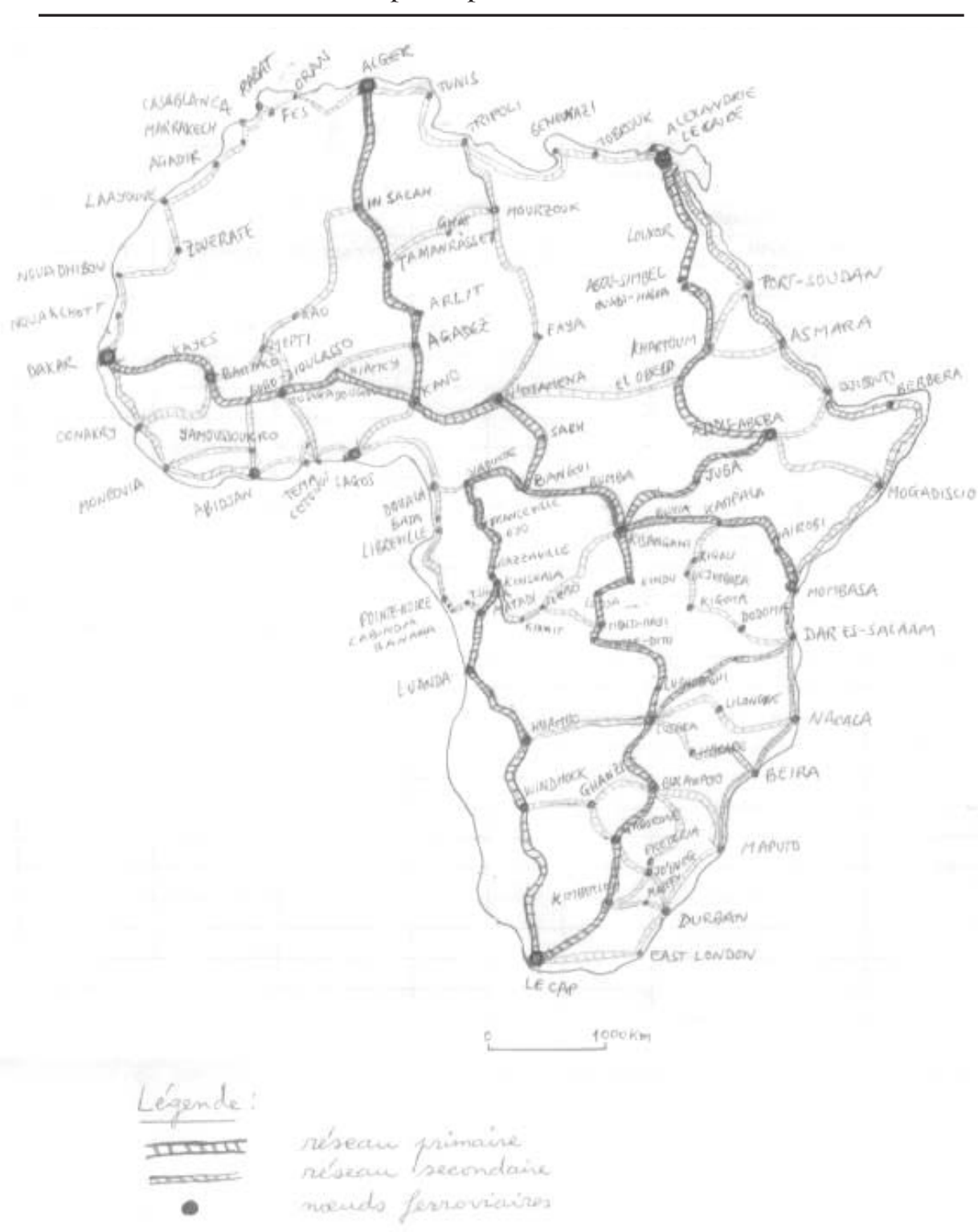

